\title{
LDR Image for Suitable Display in Conventional Display Devices by CIELAB based Tone-Mapping Algorithm
}

\author{
Sadaf Afreen \\ M. Tech Scholar \\ All Saints College of technology \\ Bhopal M.P. India
}

\author{
Aizaz Tirmizi \\ Assistant Professor \\ All Saints College of technology \\ Bhopal M.P. India
}

\begin{abstract}
This paper presents a analysis of the CIELAB color feature based tone mapping technique. After analysis of these techniques we had concluded that saliency based tone mapping algorithm is not computationally efficient as good as the proposed methodology. The different Salience-based Tone mapping method for High dynamic range images the halo artifacts significantly reduced. The visual quality of tonemapped image, especially attention-salient regions, is improved by the saliency-aware weighting. Experimental results show that the saliency-aware technique and the proposed method produce good results on a variety of high dynamic range images. The Visual quality of the proposed method is same as of saliency based tone mapping but it is more computational efficient.
\end{abstract}

\section{Keywords}

Edge-aware weighting, high dynamic range (HDR), local filtering, saliency-aware weighting, tone mapping.

\section{INTRODUCTION}

We experience in our daily life that the real world scenes often have a very wide range of luminance values. Quality of the image can be improved with the concept of lots of images of same object at the same position can be taken as the raw images. Although it would be possible to capture high dynamic range (HDR) photos with the future digital cameras. In any one single shot only a part of the real world high dynamic scene is visible with the current technology. Such a scenario is illustrated in Figure (1). It is an indoor scene with the sunlight coming through the window and the camera was placed at the dark end. To make the features visible near the window, less exposure was used. However, this made the scene further away from the light source too dark [1]. We increased the exposure interval to make the features visible in the dark end. To human observers, however, all features in the darkest as well as the brightest areas are equally clearly visible simultaneously. In fact, recent technologies have made it relatively easy to create numerical luminance maps that capture the full dynamic range of real world scene [1], [2].For a sequence of low dynamic range (LDR) images of the same scene taken under different exposure levels, a HDR radiance map of the scene can be generated. Several works try to select the most appropriate LDR images to generate the HDR image where many LDR images with various exposures are already captured and stored; it implies that larger storage, as well as higher energy consumption is needed for such scenarios.

In this paper, we advocate an alternative edge-preserving operator, based on the weighted least squares (WLS) framework. This framework was originally used to reduce ringing while deblurring images in the presence of noise [Lagendijk et al. 1988], and it is closely related to biased anisotropic diffusion [Nordstrom 1989]. Recently, this framework was employed for piecewise smooth propagation of sparse constraints [Levin et al. 2004; Lischinski et al. 2006]. We show that the WLS-based operator is robust and versatile, and may be used in many of the applications that have so far been based on the BLF, at the cost of longer computation times. We found this operator to be particularly well-suited for progressive coarsening of images, and for extraction of detail at various spatial scales. Thus, we use it to construct a new kind of an edge-preserving multi-scale image decomposition, which provides an excellent foundation for multi-scale HDR and LDR tone mapping, detail enhancement, and contrast manipulation.

The remainder of this paper is organized as follows. In the next section we discuss various applications of base-detail decompositions, explain the causes of common artifacts, and examine the characteristics of the bilateral filter in this context. Next, in Section 3 we show how the WLS framework may be used to perform edge preserving smoothing, and describe the multi-scale decomposition construction process. Section 4 presents a detailed comparison between our WLSbased multi-scale decompositions with several previous schemes, while Section 5 discusses its connections with spatially-variant filtering and with anisotropic diffusion. Finally, we demonstrate the utility of our decompositions in the context of several applications.

\section{BACKGROUND OF PROPOSED METHOD}

In computational photography, images are often decomposed into a piecewise smooth base layer and one or more detail layers. The base layer captures the larger scale variations in intensity, and is typically computed by applying an edgepreserving smoothing operator to the image (sometimes applied to the logarithm of the luminance or to the lightness channel of the CIELAB color space). The detail layer is then defined as the difference (or the quotient) between the original image and the base layer. Each of the resultant layers may be manipulated separately in various ways, depending on the application, and possibly recombined to yield the final result. Computing the base layer is an image coarsening process. The coarsening must be done carefully in order to avoid artifacts that might arise once the base and the detail layers are manipulated separately and recombined. The ideal edgepreserving filter must neither blur nor sharpen the edges that separate coarse scale image features, while smoothing the regions between such edges. Unfortunately, such an operator does not exist, because in general it is impossible to unambiguously determine which edges should be preserved. Furthermore, in order to produce multi-scale base-detail decompositions, the operator must allow increasingly larger image features to migrate from the base layer to the detail 
layer. In other words, it must allow increasingly larger regions to become increasingly smoother.

\subsection{Edge-Preserving Smoothing via WLS}

In this section, we first describe an alternative edgepreserving smoothing approach based on the WLS optimization framework, and then show how to construct multi-scale edge-preserving decompositions that capture details at a variety of scales. Edge-preserving smoothing may be viewed as a compromise between two possibly contradictory goals. Given an input image g, we seek a new image $\mathrm{u}$, which, on the one hand, is as close as possible to $\mathrm{g}$, and, at the same time, is as smooth as possible everywhere, except across significant gradients in g. Formally, this may be expressed as seeking the minimum of

$$
\sum_{\mathrm{p}}\left(\mathrm{u}_{\mathrm{p}}-\mathrm{g}_{\mathrm{p}}\right)^{2}+\lambda\left(\mathrm{a}_{\mathrm{x}, \mathrm{p}}(\mathrm{g})\left(\frac{\partial \mathrm{u}}{\partial \mathrm{x}}\right)^{2}+\mathrm{a}_{\mathrm{y}, \mathrm{p}}(\mathrm{g})\left(\frac{\partial \mathrm{u}}{\partial \mathrm{y}}\right)^{2}\right)(1)
$$

where the subscript $\mathrm{p}$ denotes the spatial location of a pixel. The goal of the data term $\left(u_{p}-g_{p}\right)^{2}$ is to minimize the distance between $\mathrm{u}$ and $\mathrm{g}$, while the second (regularization) term strives to achieve smoothness by minimizing the partial derivatives of $u$. The smoothness requirement is enforced in a spatially varying manner via the smoothness weights ax and ay, which depend on g. Finally, 1 is responsible for the balance between the two terms; increasing the value of 1 results in progressively smoother images $u$. where the subscript $p$ denotes the spatial location of a pixel. The goal of the data term $\left(u_{p}-g_{p}\right)^{2}$ is to minimize the distance between $u$ and $g$, while the second (regularization) term strives to achieve smoothness by minimizing the partial derivatives of $u$. The smoothness requirement is enforced in a spatially varying manner via the smoothness weights ax and ay, which depend on $\mathrm{g}$. Finally, $\lambda$ is responsible for the balance between the two terms; increasing the value of 1 results in progressively smoother images $\mathrm{u}$.

$$
\mathrm{a}_{\mathrm{x}, \mathrm{p}}(\mathrm{g})=\left(\left|\frac{\partial \mathrm{l}}{\partial \mathrm{x}}(\mathrm{p})\right|^{\alpha}+\varepsilon\right)^{-1} \mathrm{a}_{\mathrm{y}, \mathrm{p}}(\mathrm{g})=\left(\left|\frac{\partial \mathrm{l}}{\partial \mathrm{y}}(\mathrm{p})\right|^{\alpha}+\varepsilon\right)^{-1}
$$

where 'is the log-luminance channel of the input image g, the exponent a (typically between 1.2 and 2.0) determines the sensitivity to the gradients of $\mathrm{g}$, while $\mathrm{e}$ is a small constant (typically 0.0001) that prevents division by zero in areas where $\mathrm{g}$ is constant. It should be noted that since the smoothness coefficients in above equation, separate between gradients in the $\mathrm{x}$ and $\mathrm{y}$ directions, the resulting operator is not rotationally invariant, with a slight tendency to preserve axis aligned edges more than diagonal ones.

\subsection{Multi-Scale Edge-Preserving Decompositions}

Using the edge-preserving operator described above, it is easy to construct a multi-scale edge-preserving decomposition, fashioned after the well-known Laplacian pyramid [Burt and Adelson 1983]. The decomposition consists of a course, piecewise smooth, version of the image, along with a sequence of difference images, capturing detail at progressively finer scales. More specifically, let $g$ denote the input image for which we would like to construct a $(\mathrm{k}+1)$ level decomposition. Let $u^{1} ; \ldots ; u^{k}$ denote progressively coarser versions of $\mathrm{g}$. The coarsest of these versions, $u^{k}$ will serve as the base layer $\mathrm{b}$, with the $\mathrm{k}$ detail layers defined as $d^{1}=u^{i-1}-u^{i}$ where $i=1, \ldots, k$ and $u^{0}=g$. the original image $g$ is easily recovered from this decomposition by simply adding up the base and the detail layers:

$$
g=b+\sum_{i=1}^{k} d^{i}
$$

Note that we do not perform any downsampling of the smoothed images $u_{i}$, since they are obtained via edgepreserving smoothing and are not band-limited in the usual sense. Thus, our multi-scale decomposition is an overcomplete description of the input image.

\subsection{Multi-Scale Tone Manipulation}

We have implemented a simple interactive tool for manipulating the tone and contrast of details at different scales. Given an image, we first construct a three-level decomposition (coarse base level $\mathrm{b}$ and two detail levels $d 1 ; d 2$ ) of the CIELAB lightness channel. This is done using the first (non-iterative) construction given by eq. (3). The user is then presented with a set of sliders for controlling the exposure $\mathrm{h}$ of the base layer, as well as the boosting factors, $\mathrm{d} 0$ for the base, and $\mathrm{d} 1 ; \mathrm{d} 2$ for the medium and fine detail layers. The result of the manipulation $\hat{g}$ at each pixel $\mathrm{p}$ is then given by -

$$
\hat{g}_{p}=\uparrow \mu+S\left(\delta_{0} \eta b_{p}-\mu\right)+S\left(\delta_{1} d_{p}^{1}\right)+S\left(\delta_{2} d_{p}^{2}\right)
$$

Where $\mathrm{m}$ is the mean of the lightness range, and $\mathrm{S}$ is a sigmoid curve,

$$
S(a ; x)=1=(1+\exp (-a x))
$$

(appropriately shifted and normalized). The goal of this sigmoid is to avoid the hard clipping that would otherwise occur when the detail layers are significantly boosted. The term $\mathrm{S}(\delta 0 ; \eta \mathrm{bp}-\mu)$ controls the exposure and contrast of the base layer, while the remaining terms control the boosting of the medium and fine scale details. Note that once the decomposition has been computed, eq. (4) is evaluated in real time. We found that this simple tool is already very effective for controlling the amount of local contrast at the different scales. The effective manipulation range is very wide: it typically takes a rather extreme manipulation to cause artifacts to appear. Example results are shown in Figures 1, 8, and 9. The decomposition for all these results was constructed with the parameters $\alpha=1: 2 ; \lambda=0: 1$ for the fine scale filtering and $\alpha$ $=1: 4 ; \lambda=0: 4$ for the medium scale filtering. We found three pyramid levels to be sufficient for the images we experimented with. Using more levels would enable finer control, but would also require the user to manipulate more sliders.

\section{HDR TONE MAPPING}

Our decompositions are easily harnessed to perform detail preserving compression of HDR images. For example, we can simply replace the BLF in the tone mapping algorithm of Durand and Dorsey, with WLS-based smoothing and avoid the mild halo artifacts that are sometimes visible in their results. Another option we experimented with is to use the tone mapping algorithm proposed by Tumblin and Turk [1999], but replace their LCIS-based multi-scale decomposition with our WLS-based decomposition. Specifically, we compute a 4-level decomposition (one coarse base layer and three detail layers) of the log-luminance channel, multiply each level by some scaling factor, and reconstruct a new log-luminance channel. In the leftmost image, our goal was to achieve a rather flat image with exaggerated local contrasts (similar, but more extreme than the typical result produced with LCIS on this image). This was achieved by strongly compressing the base, and boosting the fine scale detail layer. 


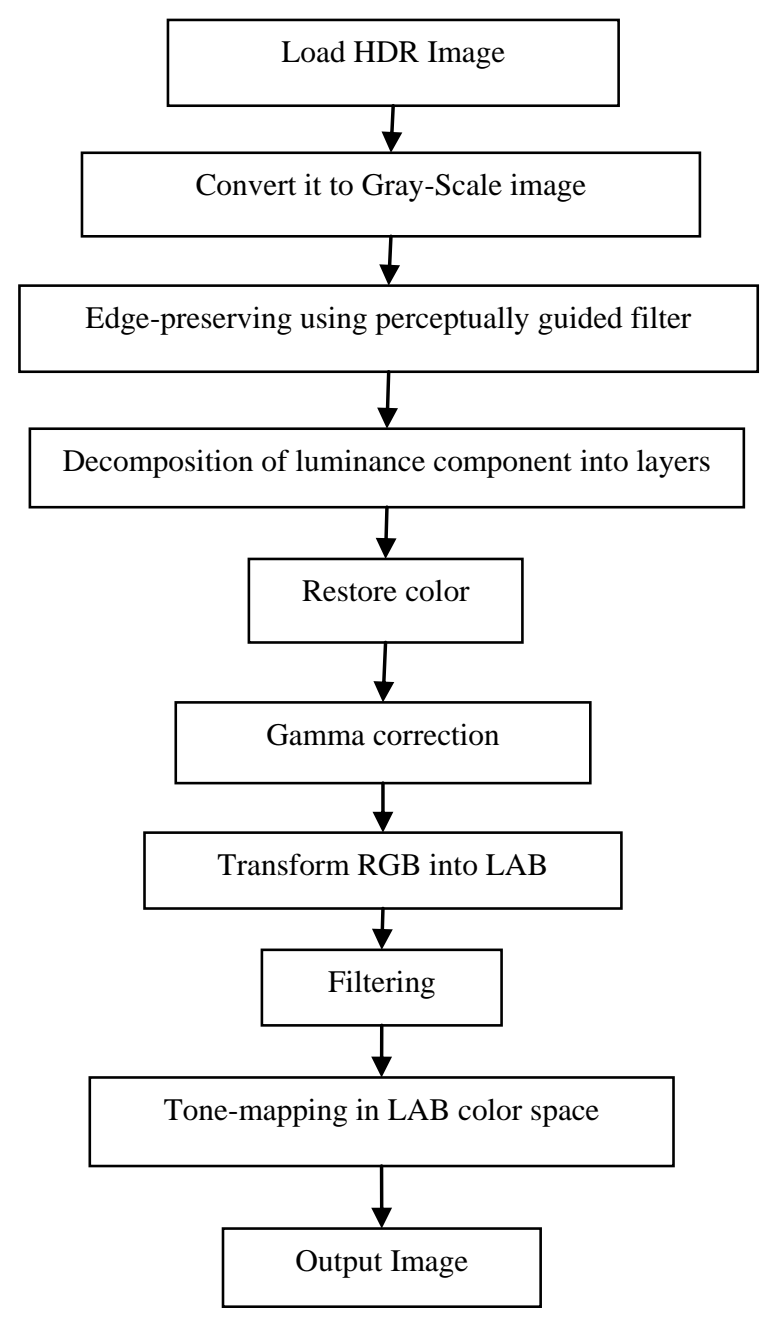

Fig.1 The Block Diagram of the Proposed Algorithm.

In the rightmost image, we pursued a more photographic look but with more depth in the highlights than what is possible with Reinhard's operator [2002]. We achieved this result by using less compression of the base and moderate boosting of the coarser detail layers.

\subsection{Progressive image abstraction}

Our WLS-based operator can be used in many other applications that utilize an edge-preserving filter. For example, Winnem"oler et al. [Winnem"oller et al. 2006] and Chen et al. [2007] use the BLF for image and video abstraction.

$$
u^{i+1}=F_{c^{i} \lambda}\left(u^{i}\right)
$$

$F_{\lambda}$ a nonlinear operator, which depends on $\mathrm{g}$ is a gradient : for some initial value of $\lambda$ and some factor c. A coarsening sequence generated in this manner and the corresponding detail layers. Using our multi-scale decomposition (the iterative version of eq. (5)) produces the results. In this application, the detail layers are attenuated, rather than boosted, to achieve a stylized abstract look. Using progressively coarser decomposition levels increases the degree of abstraction in the resulting image. These abstractions can also be combined together in a spatially varying manner to provide more detail in areas of interest. We do this with an interactive painting interface; a more automated mechanism is described in [Chen et al. 2007]. The images are overlaid with edges extracted from the appropriate detail layers.

\section{EXPERIMENTAL RESULTS}

We have implemented a number of simple tools that use our multiscale edge-preserving decompositions for photographic tone manipulation, HDR tone mapping, detail enhancement, and image abstraction. Below, we briefly describe these tools and show some results. Note the purpose of these tools is to demonstrate in the simplest possible way the robustness and versatility of our decompositions.

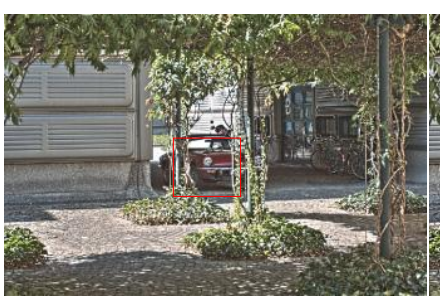

(a)

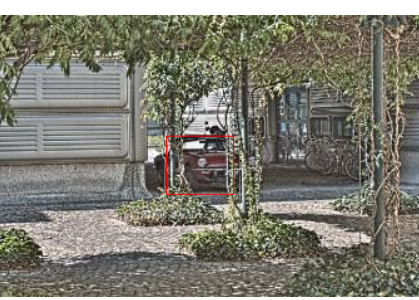

(b)

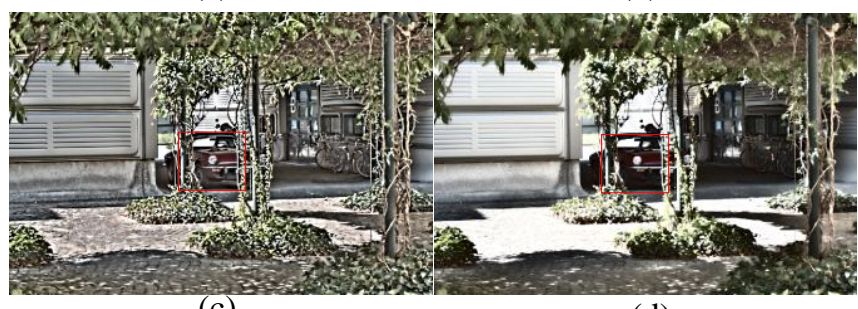

(c)

(d)
Fig.2 (a) Result image of the Saliency based tone mapping and (b), (c) and (d) Result image of the propose tone mapping algorithm with fine, medium and coarse detail respectively.

Any of these tools could have been made much more sophisticated, but this is outside the scope of this paper. In this application, the detail layers are attenuated, rather than boosted, to achieve a stylized abstract look.

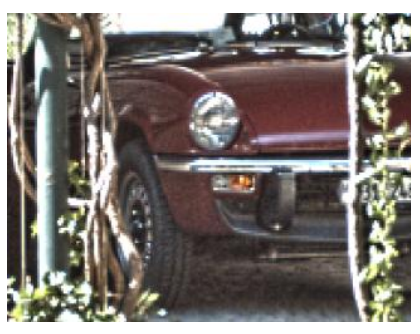

(a)

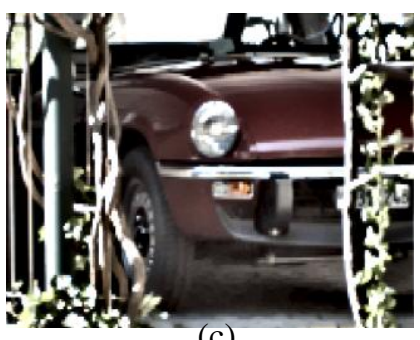

(c)



(b)

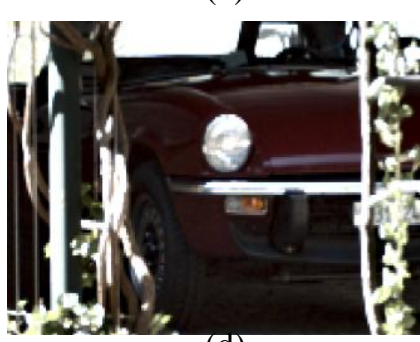

(d)
Fig.3 Zoom of Result Image from fig 2 with proper visibility (a) Result image of the Saliency based tone mapping and (b), (c) and (d) Result image of the propose tone mapping algorithm with fine, medium and coarse detail respectively. 
Using progressively coarser decomposition levels increases the degree of abstraction in the resulting image. These abstractions can also be combined together in a spatially varying manner to provide more detail in areas of interest.

\section{CONCLUSION AND DISCUSSION}

Multi-scale contrast manipulation is a valuable digital darkroom technique. Our results on a variety of applications, including tone mapping, contrast manipulation, and image abstraction, show that our approach is robust and versatile. In future work we would like to investigate more sophisticated schemes for determining the smoothness coefficients for the WLS formulation in order to further improve the ability to preserve edges and extract details. Another important issue that must be tackled is better handling of color. Our multiscale tone manipulation tool currently operates on the CIELAB lightness channel, and we have observed that strong manipulations result in significant changes in the perceived color.

\section{ACKNOWLEDGMENTS}

Our special thanks to the experts who have contributed towards the development of this paper.

\section{REFERENCES}

[1] P. E. Debevec and J. Malik, "Rendering high dynamic range radiance maps from photographs," in Proc. SIGGRAPH, Los Angeles, CA, USA, Aug. 1997, pp. 369-378.

[2] J. Tsotsos, "Analyzing vision at the complexity level," Behav. Brain. Sci., vol. 13, no. 3, pp. 423-445, Mar. 1990.

[3] A. Borji, D. N. Sihite, and L. Itti, "Quantitative analysis of human model agreement in visual saliency modeling: A comparative study," IEEE Trans. Image Process., vol. 22, no. 1, pp. 55-69, Jan. 2013.

[4] S. Grgic, M. Grgic, and B. Zovko-Cihlar, "Performance analysis of image compression using wavelets," IEEE Trans. Ind. Electron., vol. 48, no. 3, pp. 682-695, Jun. 2001.

[5] P. Y. Hsiao, C. L. Lu, and L. C. Fu, "Multilayered image processing for multiscale Harris corner detection in digital realization," IEEE Trans. Ind. Electron., vol. 57, no. 5, pp. 1799-1805, May 2010
[6] J. Li, Y. H. Tian, T. J. Huang, and W. Gao, "Probabilistic multi-task learning for visual saliency estimation in video," Int. J. Comput. Vis., vol. 90, no. 2, pp. 150-165, Nov. 2010

[7] S. Y. Chen, G. J. Luo, X. Li, S. M. Ji, and B. W. Zhang, "The specular exponent as a criterion for appearance quality assessment of pearl like objects by artificial vision," IEEE Trans. Ind. Electron., vol. 59, no. 8, pp. 3264-3272, Aug. 2012.

[8] C. Y. Chang and H. Lie, "Real-time visual tracking and measurement to control fast dynamics of overhead cranes," IEEE Trans. Ind. Electron., vol. 59, no. 3, pp. 1640-1649, Mar. 2012.

[9] H. L. Zhuang, K. S. Low, and W. Y. Yau, "Multichannel pulse-coupled neural network-based color image segmentation for object detection," IEEE Trans. Ind. Electron., vol. 59, no. 8, pp. 3299-3308, Aug. 2012.

[10] J. Garcia et al., "Directional people counter based on head tracking," IEEE Trans. Ind. Electron., vol. 60, no. 9, pp. 3991-4000, Sep. 2013.

[11] E. Reinhard, G. Ward, S. Pattanaik, and P. E. Debevec, High Dynamic Range Imaging: Acquisition, Display and Image-Based Lighting. San Mateo, CA, USA: Morgan Kaufmann, 2005

[12] R. Fattal, D. Lischinski, and M.Werman, "Gradient domain high dynamic range compression," ACMTrans. Graphics, vol. 27, no. 3, pp. 67:1-67:10, Jul. 2002.

[13] E. Reinhard, M. Stark, P. Shirley, and J. Ferwerda, "Photographic tone reproduction for digital images," ACM Trans. Graphics, vol. 21, no. 3, pp. 267-276, Jul. 2002.

[14] A. Adams, The Print. ser. The Ansel Adams Photography series. New York, NY, USA: Little, Brown and Company, 1983.

[15] Z. Farbman, R. Fattal, D. Lischinski, and R. Szeliski, "Edge-preserving decompositions for multi-scale tone and detail manipulation," ACM Trans. Graphics, vol. 21, no. 3, pp. 249-256, Aug. 2008.

[16] Zhengguo Li and Jinghong Zheng "Visual-SalienceBased Tone Mapping for High Dynamic Range Images" IEEE Transactions on Industrial Electronics, Vol. 61, No. 12, December 2014 